\title{
Le français, langue de scolarisation: vers une didactique spécifique
}

\section{Michel Verdelhan, Bruno Maurer et Marie-Claire Durand}

\section{(2) OpenEdition}

1 Journals

Édition électronique

URL : http://journals.openedition.org/trema/1758

DOI : $10.4000 /$ trema. 1758

ISSN : 2107-0997

Éditeur

Faculté d'Éducation de l'université de Montpellier

\section{Édition imprimée}

Date de publication : 1 octobre 1999

Pagination : 147-157

ISSN : 1167-315X

\section{Référence électronique}

Michel Verdelhan, Bruno Maurer et Marie-Claire Durand, « Le français, langue de scolarisation: vers une didactique spécifique », Tréma [En ligne], 15-16| 1999, mis en ligne le 01 octobre 1999, consulté le 23 avril 2019. URL : http://journals.openedition.org/trema/1758; DOI : 10.4000/trema.1758

Ce document a été généré automatiquement le 23 avril 2019.

Trema 


\title{
Le français, langue de scolarisation: vers une didactique spécifique
}

\author{
Michel Verdelhan, Bruno Maurer et Marie-Claire Durand
}

Après avoir situé cette notion dans la réflexion didactique sur le français langue seconde nous proposerons quelques éléments d'analyse avec le souci de faire apparaître l'intérêt pédagogique et les conséquences méthodologique de cette fonction de scolarisation. Une série de composantes du français de scolarisation concerne l'accès au savoir l'autre concerne la socialisation. Ainsi notre sujet de travail dans cet atelier est-il bien au cœur des préoccupations du colloque.

2 Marie-Claire Durand prolongera notre analyse du français de scolarisation en s'intéressant au double jeu de la langue écrite, objet d'apprentissage et outil d'apprentissage.

3 Bruno Maurer, reviendra sur l'aspect social de la langue de scolarisation et les conséquences didactiques qui en découlent.

\section{Le français de scolarisation. Michel Verdelhan}

\subsection{L'origine de la notion}

On rencontre la notion de français de scolarisation quand on travaille dans le domaine de la didactique du français langue seconde.

5 Le français langue seconde renvoie à tout un ensemble de situations dans lesquelles le français n'est pas simplement une langue étrangère. Les sociolinguistes décrivent ces situations ou le français est tantôt la langue de l'administration, tantôt la langue officielle ou l'une des langues officielles ou langue nationale, langue de certains secteurs d'activités, et langue de l'école. Ces situations ont bien sûr une explication historique : pour beaucoup, il s'agit de l'héritage de la période coloniale.

6 En soulignant l'extrême diversité de ces cas, les sociolinguistes semblent rendre impossible la conception d'une méthodologie générale du Français Langue Seconde (FLS) 
comme on a une méthodologie du Français Langue Etrangère (FLE). Cette diversité semble condamner la recherche méthodologique à des solutions locales spécifiques... Et c'est d'ailleurs ce qui souvent s'est produit.

\subsection{La notion de français de scolarisation en FLS}

7 Si l'on cherche ce que les situations de FLS ont en commun, le P.G.C.D qui s'impose est ce rôle général du français comme langue de scolarisation.

Cette fonction de scolarisation est présente dans tous les cas repérés de FLS avec des variantes. Ces variantes concernent le moment dans la scolarité où débute cette fonction et son extension à quelques disciplines ou à toutes. Mêmes les filières bilingues développées sous l'égide de l'Agence Universitaire de la Francophonie redonnent au français cette fonction de scolarisation. Ainsi par exemple, en Haïti, l'élève est scolarisé d'abord en créole puis en français.

Lire est un jeu d'enfant La lecture est un plaisir pour la vie, auquel on peut initier l'enfant dès le berceau.

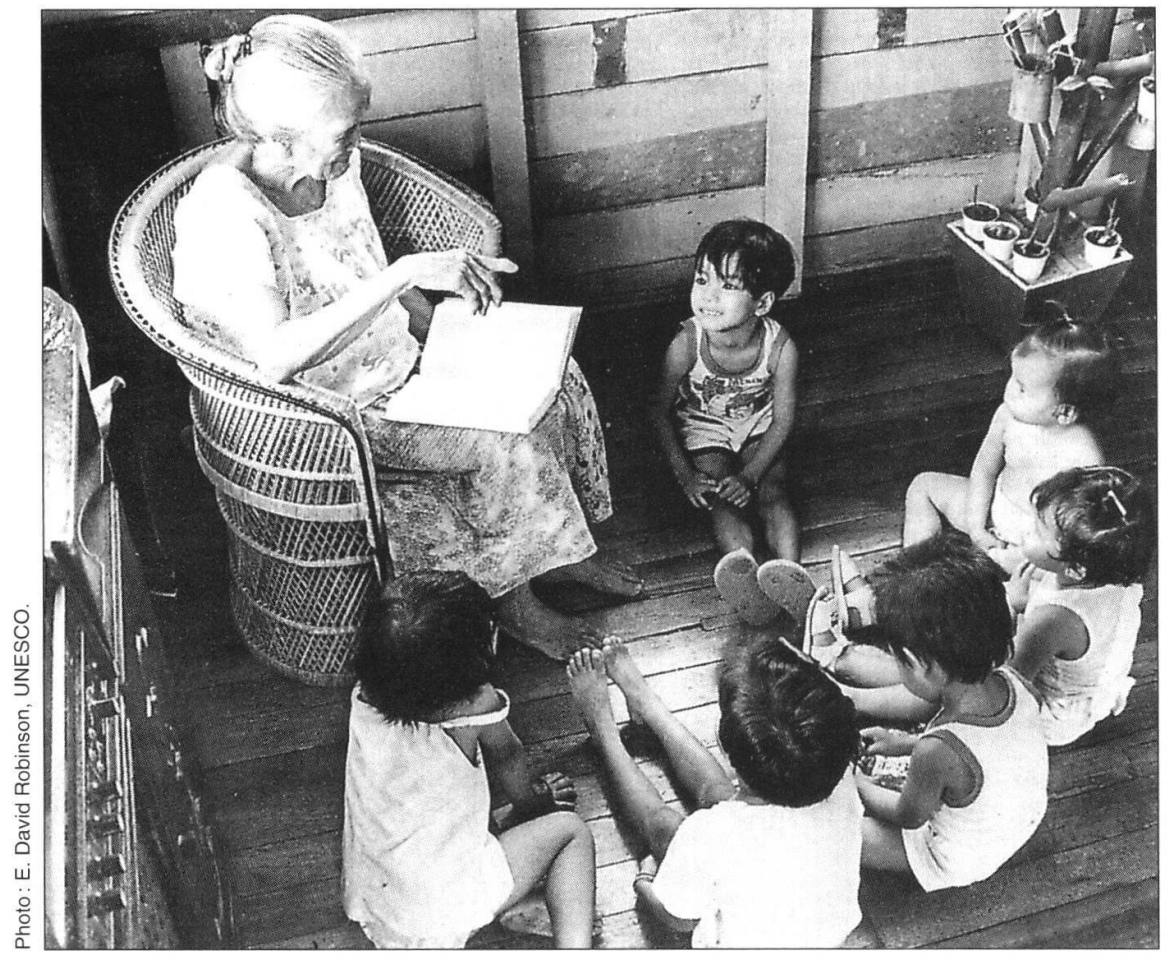

Le Courrier de I'UNESCO. Paris, UNESCO, Juillet 1990 (Vol. 43, №7), 50 p., p. 37.

$\mathrm{Au}$ Vietnam dans les filières francophones, on apprend d'abord à lire en vietnamien puis on apprend le français et, progressivement, des disciplines sont enseignées en français (math et sciences).

Par cette fonction de scolarisation, le français langue seconde retrouve le rôle du français en situation de langue maternelle. (F.L.M.) 


\subsection{La notion de français de scolarisation en FLM}

9 En français langue maternelle, le français est but et moyen d'enseignement de lui même et des autres disciplines. Ce statut est souvent mentionné dans les I.O. En particulier pour l'Ecole primaire, on rappelle souvent que le français sert à toutes les disciplines et que toutes les disciplines concourent à son enseignement. Les I.O de 1972 rappelaient que «l'instituteur enseigne constamment le français ». Les I.O de 1995 pour la même idée parlent de compétence transversale.

10 On ne peut tirer les conséquences de cette banalité sans passer par l'analyse de cette fonction générale de scolarisation.

\subsection{L'analyse de la fonction de scolarisation}

11 L'expression "le français de l'école» ou celle de la "langue des messieurs" pour reprendre le titre de P. Boutan, oriente la recherche vers l'analyse des écarts entre la langue utilisée à l'école et celle des enfants. C. Vargas, dans ce sens, parle de «Français norme étrangère par rapport aux normes identitaires des enfants...!». Mais c'est une autre orientation que celle proposée ici.

La didactique du français langue seconde a repéré quelques composantes de la fonction de scolarisation. Ces composantes se retrouvent en F.L.M et elles suggèrent des solutions pédagogiques.

On peut lister, d'une part, des composantes liées à l'accès au savoir et, d'autre part, des composantes liées à la communication scolaire.

Sans prétendre épuiser l'analyse et à partir de la situation d'enseignement du F.L.S, on distingue aisément dans le rôle de médiation au savoir quatre grandes fonctions :

Une fonction d'exposition du savoir

En F.L.S, les professeurs font des cours, présentent des connaissances en français...

13 Une fonction de concentration du savoir

A l'école primaire, la "trace écrite », comme le résumé concentre le savoir (Cf., les cartouches et encadrés, les rubriques « je retiens » ou « à retenir » des manuels).

Une fonction instrumentale de construction du savoir

Le français accompagne et rend possible le travail de la pensée qui soutient l'appropriation des connaissances.

15 Une fonction d'exercitation

A l'école pour apprendre, on s'entraîne, on fait des exercices, on exécute des consignes...

\section{Les composantes de la fonction de scolarisation et la pédagogie du français}

16 L'identification de ces composantes conduit à se demander si l'enseignement du français se préoccupe suffisamment de donner la maîtrise de la langue dans ces fonctions. 
Fac-similé d'une fiche scolaire de la République de Djibouti.

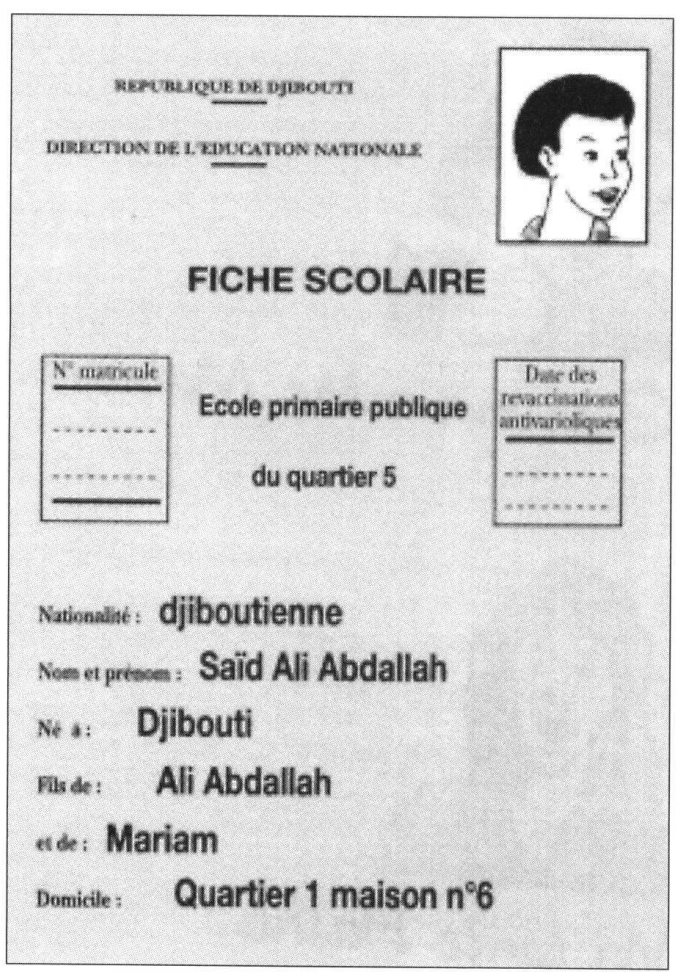

HASSAN Z.H., CORNETTE Y., ROBERT J.-P. et MAURER B. : Le Cabri et le Chacal - Livre de français Cours d'Initiation. Vanves, CRIPEN-Djibouti (Centre de Recherche d'Information et de Production de l'Éducation Nationale) - EDICEF, 1993.127 p., p. 18.

\subsection{La fonction d'exposition et la pédagogie de la compréhension}

En F.L.S., pour tirer profit d'un cours fait en français, l'élève doit comprendre l'exposé du professeur. Malgré l'inévitable présence des mots et tournures qu'il ne connaît pas, puisque le français n'est pas sa langue maternelle, il faut qu'il comprenne le cours. S'il se laisse piéger par ces éléments inconnus, s'il laisse son attention captée par la part de système linguistique, il va perdre le reste de l'exposé.

En F.L.M., l'élève doit souvent surmonter la même difficulté et éviter le même piège. A-ton suffisamment pensé à apprendre et comprendre? Est-ce qu'une pédagogie de la compréhension orale est justifiée en F.L.M. comme en F.L.S. ? Quels exercices seraient possibles? Une pédagogie de la compréhension orale ne se réduit pas à l'ensemble des procédés utilisés par les maîtres pour essayer d'assurer la compréhension de ce qu'ils disent. Pas plus qu'une pédagogie de la compréhension écrite ne peut être confondue avec les notes qui expliquent les mots d'un texte.

\subsection{La concentration du savoir et la double pédagogie du résumé}

19 Les dernières Instructions Officielles pour l'école élémentaire préconisent une pédagogie du résumé avec une progression qui commencerait dès le cycle 1. Cette préoccupation précoce est bien évidemment justifiée par la présence de l'activité résumante dans l'acte de lire. 
Mais, pour être utile, le savoir qui a été concentré pour être mémorisé doit pouvoir ensuite être "déconcentré ». Les phrases du résumé des leçons constituent le texte à partir duquel le savoir doit pouvoir être retrouvé. Et finalement, l'élève doit être capable de concentrer le savoir et de l'exposer.

Jusqu'ici notre enseigne s'est attachée davantage à apprendre à résumer qu'à réaliser l'opération inverse. Comment apprendre à développer du savoir après avoir appris à le ramasser?

\subsection{La fonction d'instrumentation dans la construction du savoir}

Cette fonction apparait notamment dans le cheminement des reformulations en science. Le passage de la conceptualisation qui suivent la réflexion.

En F.L.S., le problème se pose dès le premier niveau lors de la formulation de l'expérience commune, dès l'expression du résultat des premières observations.

On explique dans notre jargon pédagogique que l'enseignant doit d'abord «faire émerger les représentations ». Mais le professeur, en F.L.S., sait que les moyens linguistiques utiles pour cette expression préscientifique vont manquer.

Est-on sûr qu'en langue maternelle, tous les élèves disposent des moyens linguistiques pour dire leurs observations? Comment en science, donner la maîtrise du français utile pour faire des sciences. Abstraction, généralisation mobilisent des matériaux linguistiques particuliers: déterminant défini, présent de vérité générale, lien de causalité...

Dire que l'instituteur enseigne le français dans toutes les disciplines reste incantatoire si l'on n'en tire pas de conséquences pratiques.

\subsection{La fonction « d'exercitation » et la pédagogie de la consigne}

Pour être fixé et approfondi tout savoir a besoin d'exercices qui passent par des consignes.

En F.L.S. une difficulté de compréhension littérale de la consigne peut venir s'ajouter aux difficultés de l'exercice et obscurcir totalement la tâche à réaliser. Les consignes exigent des tâches sans révéler les conduites à tenir. Aux différents niveaux de scolarité en F.L.M. et récemment en F.L.S. on trouve des gammes d'activités visant la maîtrise de la lecture des consignes.

Une pédagogie de la consigne est peut-être en train de se constituer. Par ses réflexions dans le cadre de sa recherche, Marie-Claire Durand y contribue.

\section{De la langue écrite objet d'apprentissage à la langue écrite outil d'apprentissage. Marie-Claire Durand}

\subsection{Le français de scolarisation et la transmission des savoirs}

Le français de scolarisation ne doit pas être considéré comme une langue spécifiquement scolaire qui serait coupée de la vie et de la société. Cette langue de scolarisation appartient certes à la culture scolaire mais elle même fait partie de la culture de notre société. En effet, l'école est un sous-système de notre système social ; une fonction est la transmission de la culture aux jeunes générations. C'est au service de cette fonction que 
sont élaborées des stratégies et des procédures d'enseignement et que sont définis les savoirs à transmettre. La langue de scolarisation, pour assurer la transmission des savoirs et de la culture de la société, se dote ainsi de genres textuels qui leur sont propres.

\subsection{Les écrits du français de scolarisation} savoirs et facteur déterminant dans leur construction par l'apprenant.

Ce double rôle apparaît dans certains genres textuels, les consignes et les énoncés d'exercices notamment.

Mon travail de recherche porte sur les problèmes linguistiques et psycholinguistiques posés par la lecture et la compréhension des consignes et des énoncés d'exercices. Des observations conduites dans des classes «ordinaires» du cycle 3 de l'école primaire et dans des classes «spéciales » de collège pour élèves en grande difficulté autorisent à penser que ce type de textes est peut-être insuffisamment pris en compte par les enseignants qui n'engagent pas les élèves à construire des savoirs à leur propos.

Les enseignants n'ont probablement pas tiré toutes les conséquences de l'importance d'une part de ces savoirs pour la lecture et la compréhension des textes et d'autre part de leur nécessaire mobilisation lors des activités mentales qui doivent être conduites pour exécuter les consignes. Pour donner aux élèves la maîtrise des textes prescriptifs consignes et énoncés d'exercices - il faut donc développer chez eux la compétence discursive textuelle correspondante.

\subsection{Une compétence discursive textuelle en français de scolarisation}

L'acquisition de cette compétence discursive textuelle passe par la construction de connaissances sur les genres textuels présents dans les exercices scolaires. Ces connaissances concernent la superstructure de ces textes ainsi que les faits de langue mobilisés.

Ainsi, pour les consignes et les énoncés d'exercice, il est important de savoir distinguer :

- la partie informative de la partie prescriptive

- les informations utiles et les informations inutiles pour la réalisation des problèmes ;

- les questions intermédiaires qui balisent la tâche et la question essentielle qui en fixe le but.

Les faits de langue, eux, sont porteurs d'indices importants à repérer (adverbe de temps, mots de coordination, signes de ponctuation, indication de nombre...).

Evidemment, pour ce travail réflexif et ces observations, la stabilité de l'écrit est indispensable.

\subsection{Scolarisation et outils d'aide à l'apprentissage}

En construisant des connaissances sur ces textes, on facilite la lecture et la compréhension et l'on rend possible l'élaboration d'outils d'aide à l'apprentissage. Ces outils se construisent grâce au développement d'activités cognitives de haut niveau: activités du raisonnement logique, d'analyse et de synthèse. 
Ainsi, la capacité à lire les consignes et les énoncés d'exercices, mobiliser les opérations mentales pour le traitement du contenu : analyses, tris et organisation de l'information. Ces opérations mentales activent dans la mémoire de travail des savoirs sur le monde, des schémas conceptuels des procédures de résolution de problème.

Maîtriser la parole, c'est maîtriser la démocratie.

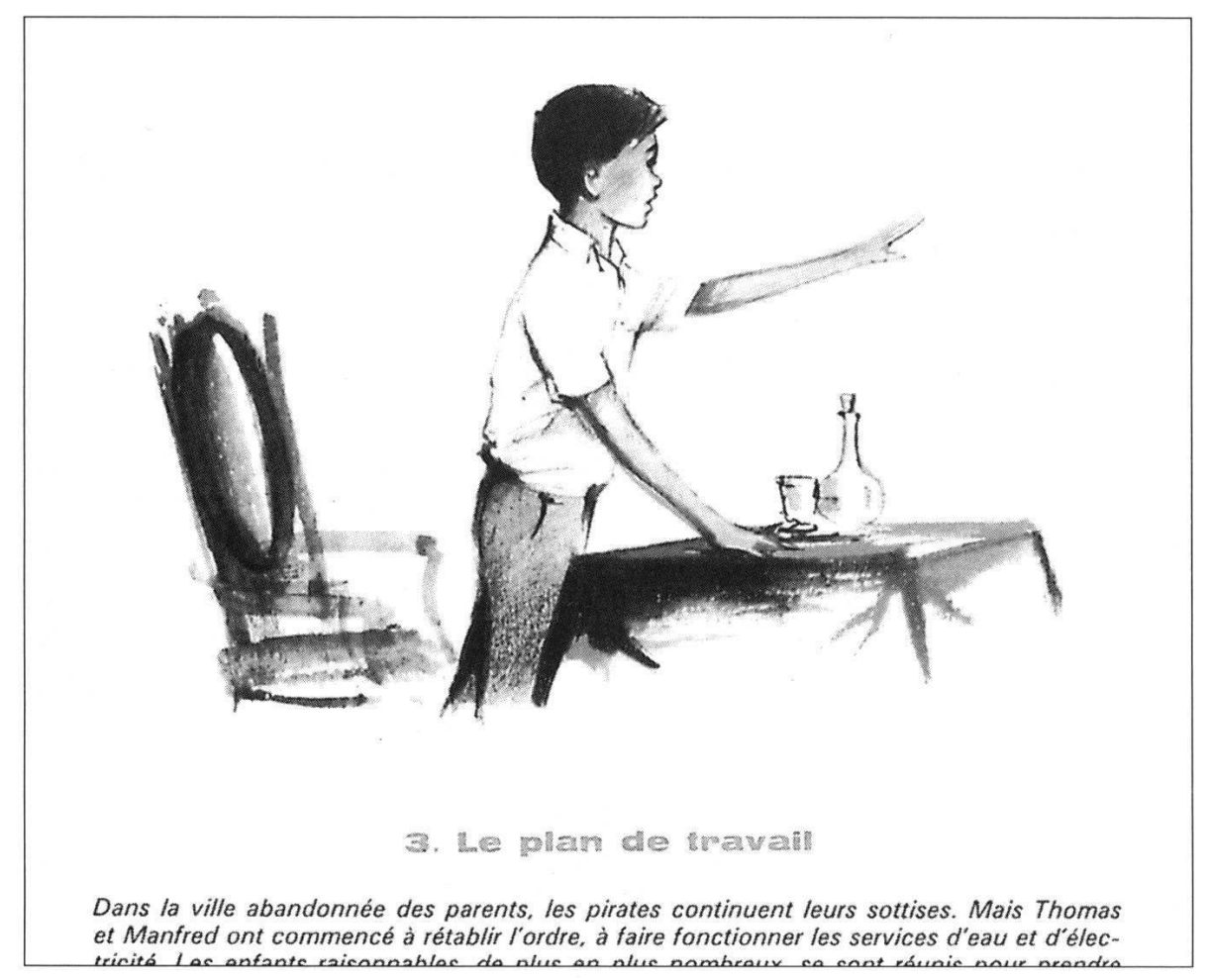

DELPIERRE P. (Inspecteur D.E.P.) et FURCY P. (Directeur d'École d'Application) : Lire et Parler - CM1. Paris, Fernand Nathan, 1967, 224 p., p. 218. Illustrations : JEAN RESCHOFSKY.

Et finalement ce sont ces activités qui fournissent aux élèves des outils par leurs apprentissages. Mais avec ces outils, on donne aussi aux élèves une compétence de communication et les moyens de l'autonomie qui rendent acteurs, responsables et citoyens.

La réussite de l'écolier dépend de la construction des outils mobilisables pour opérer les réajustements éventuellement nécessaires lors de la résolution des tâches (essais/ erreurs).

L'activité réflexive qu'autorise la permanence du texte écrit implique cette mise à distance dont bénéficie l'apprentissage du débat démocratique.

Or, ce type de débat semble être méconnu ou désappris par beaucoup de jeunes en difficulté. Et pourtant, le débat démocratique, qui demande le respect de la parole de l'autre et la découverte du respect par l'autre de sa propre parole, peut être un des moyens d'éviter la violence à l'école.

31 Il revient à notre collègue sociolinguiste Bruno Maurer de développer ce point de vue. 


\section{Le français langue de scolarisation, langue de socialisation. Bruno Maurer}

\subsection{Savoir faire communicatifs et socialisation}

Le français est langue de scolarisation, ce qui signifie principalement que c'est en français que les élèves vont accéder à d'autres savoirs, construire des connaissances. Mais on peut prendre la scolarisation sous son aspect social et la considérer également comme l'apprentissage de règles de comportement dont certaines sont particulières à ce milieu social, mais qui peuvent également se retrouver dans d'autres domaines de la communication, dans d'autres sphères sociales. De ce point de vue, une analyse de la communication scolaire dans le cadre de la classe montre que celle-ci est particulièrement dense en actes qui se révèleront très importants pour l'insertion sociale des individus, comme "proposer, refuser, dire qu'on est d'accord, dire qu'on n'est pas d'accord ». En effet, de l'aptitude à savoir négocier ces actes toujours délicats du point de vue des relations interpersonnelles comme des identités sociales, dépend pour beaucoup le jugement que les personnes avec lesquelles on est amené à entrer en contact vont porter sur le locuteur.

L'école est donc un milieu où l'on exerce des savoirs linguistiques, ici de l'ordre des savoir-faire communicatifs, qui vont ensuite se révéler extrêmement utiles pour la vie sociale. Pour certains élèves, il peut même s'agir du milieu où, prioritairement, ils vont non seulement exercer ces savoir-faire mais où ils vont pouvoir les construire. C'est en ce sens que l'on peut dire que la langue de scolarisation est aussi une langue de socialisation, et que les savoirs linguistiques en jeu dans la communication scolaire sont importants dans le reste de la vie sociale.

\subsection{Propositions didactiques}

Des conséquences didactiques devraient en être tirées à tous les niveaux de la scolarité pour traduire concrètement cette dimension de socialisation. Il faudrait pour commencer faire l'inventaire des actes de parole fréquents dans la vie de la classe qui ont une fonction socialisante forte, comme ceux mentionnés ci-dessus.

Il faut ensuite avoir le souci dans toutes les situations didactiques de faire véritablement communiquer les élèves, afin que les actes dont il est ici question soient mis en jeu; il s'agit en d'autres termes de susciter la parole, par la création de véritables situations de communication, plus que de faire parler les élèves, ce qui passe essentiellement par le biais de l'alternance questions / réponses. On voit très bien que ce type de position se trouve être en conformité avec les principes de la pédagogie institutionnelle, issue des recommandations de $\mathrm{C}$. Freinet, et qui visent à une socialisation par l'aménagement de réseaux de communication dans la classe qui favorisent toujours la coopération des élèves.

Mais à notre sens, cela ne suffit peut-être pas. On peut aussi concevoir l'idée qu'à partir de ces situations de communication soient mises en place des séances plus réflexives visant à la prise de conscience par les élèves des valeurs des formes linguistiques qu'ils échangent. De ce point de vue, toutes ne sont pas équivalentes, et certaines, qui s'inscrivent plutôt dans une recherche de collaboration, contribuent plus que d'autres, 
porteuses d'une charge d'agressivité importante, à la socialisation. Ainsi, on peut peutêtre par ce travail d'objectivation contribuer à la fois à une meilleure scolarisation amélioration du climat dans la classe - et à une meilleure socialisation - formation d'individus et plus seulement d'élèves aptes à se servir du langage comme d'un outil de coopération.

\section{Conclusion}

Ce rôle de scolarisation se manifeste dans les emplois du français qui servent à présenter le savoir, à le concentrer et à le mémoriser. Mais il se manifeste aussi dans des emplois qui conduisent au savoir, permettent sa découverte, sa construction et son appropriation. Ces accès au savoir passent par la communication qui peut s'analyser en actes de langage dont certains sont très denses pour l'investissement identitaire du sujet et lourds de conséquences pour le développement de la communication.

Ces fonctions, composantes du rôle général de la scolarisation, et ces actes de langage, éléments clefs de la communication, doivent faire l'objet d'une didactisation.

La notion de français langue de scolarisation oriente la réflexion vers une vision globale et complexe de l'enseignement de la langue : la langue sert à tout enseignement, elle est impliquée dans tout enseignement, tout enseignement est enseignement de la langue, dans et par ce rôle de scolarisation elle est aussi langue de socialisation...

Le risque est grand de voir la réflexion didactique s'enliser dans cette complexité. Pour éviter cet enlisement improductif, il nous paraît indispensable et légitime de chercher un ancrage sur les points forts qu'une analyse sans doute réductrice et simplificatrice permet de dégager.

En Français Langue Maternelle, comme en Français Langue Seconde, ce qui pourra finalement valider le concept de français de scolarisation ce seront les retombées pédagogiques, les applications méthodologiques de son analyse.

\section{RÉSUMÉS}

Le français est langue de scolarisation. Il remplit des fonctions d'exposition et de concentration du savoir, d'instrumentation et d'exercisation. Parallèlement, il a une fonction de socialisation dont l'analyse peut déboucher sur des solutions concrètes pour une pédagogie de la communication.

French is (in France) the language of schooling. It is the expository vehicle of knowledge as well as the means of its concentration; it is also its instrument and the means by which it is exercised. At the same rime, one of its functions is the socialization of students and the analysis of this socialisation may lead to concrete solutions for communications oriented teaching skills. 
INDEX

Mots-clés : langue de scolarisation, socialisation

Keywords : language used by the school, socialization

\section{AUTEURS}

MICHEL VERDELHAN

IUFM de Montpellier

\section{BRUNO MAURER}

Université Montpellier III

MARIE-CLAIRE DURAND

Conseiller Pédagogique, Nîmes 